\title{
De single mother para mãe a solo ou mãe solo na perspetiva da semântica de frames
}

\section{Translating Single Mother as Mãe a Solo or Mãe Solo from a Frame-Semantics Perspective}

\author{
Marília Sette CÂmara [mariliasette@gmail.com] \\ Universidade de Lisboa, Portugal \\ Maria Clotilde Almeida [maria.almeida@campus.ul.pt] \\ Universidade de Lisboa, Portugal
}

\begin{abstract}
RESUMO
O presente estudo, elaborado na perspetiva da semântica de frames, constitui uma abordagem semântico-lexical do frame single mother e das suas equivalências atuais em português europeu (PE) e brasileiro (PB), pondo em destaque a variabilidade e dinamicidade do significado linguístico. Decorre de um vasto estudo de aplicação da semântica de frames à tradução de colocações de um corpus sobre liderança no feminino (Câmara 2021), constituído a partir de um conjunto de obras sobre a temática em questão, a saber, Babcock \& Laschever (2003), Miller \& Miller (2011) e Sandberg \& Scovell (2013). Concretamente, o estudo incide sobre uma alteração de framing da colocação single mother, materializada na substituição da colocação equivalente mãe solteira por mãe a solo ou mãe solo (em PE e PB, respetivamente), em face das mudanças sociais envolvendo a construção social da maternidade, ocorridas nas últimas décadas a nível global.
\end{abstract}

\section{Palavras-chave}

Semântica de frames; colocações; single mother; mãe a solo/mãe solo; tradução interlinguística e intralinguística

\begin{abstract}
This paper, conducted from the perspective of frame semantics, is a lexical-semantic approach to the single mother frame and its current equivalents in European and Brazilian Portuguese which highlights the variability and dynamics of linguistic meaning. It stems from a comprehensive study of the application of frame semantics to the translation of collocations extracted from a corpus on female leadership (Câmara 2021), constituted from a set of works on the subject - namely, Babcock \& Laschever (2003), Miller \& Miller (2011), and Sandberg \& Scovell (2013). Specifically, this paper focuses on a reframing of the collocation single mother, materialized in the replacement of the equivalent collocation mãe solteira by mãe a solo or mãe solo (in European and Brazilian Portuguese, respectively), in the face of social changes involving the social construction of motherhood which have taken place in recent decades at a global level.
\end{abstract}

\section{KEYWORDS}

Frame semantics; collocations; single mother; mãe a solo/mãe solo; inter- and intralinguistic translation 


\section{Introdução}

A semântica de frames, que constitui um dos enfoques teóricos da Linguística Cognitiva, postula que o significado linguístico é construído de forma dinâmica e flexível, em articulação quer com a experiência física, quer com as alterações a nível sociocultural. Assim sendo, na semântica de frames, as palavras representam categorizações da experiência do falante no mundo, tendo por base o conhecimento e experiência partilhados no seio de uma comunidade linguística, num determinado momento no tempo. Em última instância, a abordagem da semântica de frames debruça-se sobre os fundamentos inerentes à criação de novas categorias lexicais na sua interpelação com outras categorias com elas relacionadas (Fillmore 2006: 374).

O presente estudo também parte do pressuposto geral de que os novos frames, materializados em novas categorias, ocorrem em sintonia com frames e categorias já presentes noutras línguas ou noutras variedades de uma mesma língua. Logo, para comprovarmos este pressuposto é necessário enveredarmos pela tradução interlinguística, neste caso, inglês-português, nas variedades do português europeu (PE) e brasileiro (PB).

Adotamos a semântica de frames para o estudo da colocação single mother visando um levantamento e tratamento da frequência desta colocação nas obras Women Don't Ask - Women and the Gender Divide, A Woman's Guide to Sucessful Negotiation e Lean In - Women, Work and the Will to Lead (Babcock \& Laschever 2003; Miller \& Miller 2011; Sandberg \& Scovell 2013, respetivamente), tendo por objetivo final o enquadramento das suas equivalências em PE e PB. Por outras palavras, buscamos esmiuçar pela via cognitiva os planos de fundo que motivam a realização linguística de single mother como uma forma de definir as bases da correspondência entre língua de partida e de chegada, nas duas variedades do português em apreço. Porém, a relação entre o plano de fundo conceitual e o primeiro plano, que aponta para a dimensão mais saliente do referente da unidade lexical, nem sempre é inequívoca, pelo que a sua tradução muitas vezes envolve perdas em um dos planos ou nessa relação, de forma que definimos o princípio da relevância de Ernst-August Gutt (2000), cuja teoria se alinha aos construtos da LC, como norteador das nossas decisões de tradução juntamente com a semântica de frames.

\section{Fundamentos da Semântica de Frames}

A visão da língua como um fenômeno construído a partir da interação entre a realidade percebida e os processos cognitivos permite direcionar o problema do significado lexical à perceção do mundo real ou das realidades associadas a determinado espaço-tempo, a determinada cultura, e não apenas a fatos linguísticos (Blanco-Carrión 2016: 167-168). É, portanto, na interface homem-mundo - ou seja, na conceitualização do mundo pela experiência humana - que encontramos as motivações por trás das categorizações da experiência realizadas na língua. Nessa linha, a semântica de frames se propõe a mapear essas categorizações, a fim de traçar o caminho da formação do sentido linguístico, buscando revelar a sua estrutura profunda pela via conceitual. 
À luz desta abordagem, as categorias realizam-se nas unidades lexicais (ULs). ${ }^{1}$ Se a cada uma destas categorias subjaz uma situação motivadora que ocorre sobre um pano de fundo de conhecimento e experiência, propõe-se que a partir destas situações possamos traçar a razão da existência das ULs e, consequentemente, o seu sentido em contexto (Fillmore 2006: 373-374). Por esta perspetiva, supõe-se que possamos investigar o sentido linguístico em sua dinamicidade e flexibilidade, ancorado na própria experiência física e sociocultural dos seres humanos.

A noção de domínio conceitual é um aspeto fundamental que perpassa as teorias inscritas sob a Linguística Cognitiva, investigando (i) o seu papel na língua como pressuposição - uma interface com a pragmática, onde a palavra ou expressão linguística carrega consigo um conhecimento que ultrapassa a superfície do texto; (ii) sua dimensão prototípica, que revela como a língua abriga valores culturais; e (iii) seu status de "pano de fundo", o conhecimento enciclopédico que integra o conhecimento linguístico. Aqui, procuraremos demonstrar como o frame, a noção desenvolvida por Charles J. Fillmore, constitui uma forma de relacionar o domínio linguístico ao conceitual que nos permite organizar a experiência dos falantes em campos estruturados da experiência e conhecimento humano, o que supomos fornecer uma via para a análise semântica lexical e, consequentemente, para a tradução.

Na semântica de frames, o significado linguístico está intimamente ligado ao domínio conceitual do falante, da comunidade linguística e dos seus textos. Postula-se, portanto, que não se pode aceder ao significado de uma UL sem conhecer o domínio conceitual em que está inserida. É nesse sentido que um frame pode ser entendido como uma estrutura organizadora da categorização linguística em interligação com o conhecimento do mundo. Pelas palavras de Fillmore, um frame seria "any system of concepts related in such a way that to understand anyone of them you have to understand the whole structure in which it fits" (2006: 373), sendo esta estrutura determinada não por categorias estáticas e objetivas, mas por protótipos:

[...] very often the frame or background against which the meaning of a word is defined and understood is a fairly large slice of the surrounding culture, and this background understanding is best understood as a prototype rather than as a genuine body of assumptions about what the world is like. (idem: 379 )

Mais precisamente, um frame pode ser definido como um sistema de categorias estruturadas de acordo com um contexto que a motiva (idem: 381), sendo este pensado em termos de protótipo, pois presume-se que não seja construído de forma idêntica por todos os falantes ou comunidades linguísticas. Se pensarmos nas diferenças entre as ULs bachelor e spinster, por exemplo, podemos perceber como o seu sentido é descritível apenas por referência às instituições nas quais se inscrevem: a do casamento e todo o sistema de valores que ele pressupõe na cultura ocidental. Este conjunto de pressuposições formam o contexto motivador destas categorias, sem o conhecimento do qual não se pode compreender o sentido destas unidades e, sem o qual, elas não teriam sequer razão de existir.

1 Fillmore utiliza o termo "unidade lexical" para referir-se a palavras e expressões com um único sentido: é o pareamento entre um vocábulo e um dos seus sentidos. Assim, cada aceção de uma palavra polissêmica seria uma unidade lexical individual, ao passo que verbos preposicionais da língua inglesa (Blanco-Carrión 2007: 72), constituídos de mais de uma palavra, por exemplo, formam uma unidade de sentido e, portanto, uma unidade lexical. Além disto, a unidade lexical é definida por Ruppenhofer et al. como “o pareamento de uma palavra com uma das suas aceções" (2016: 7). 
Ao observarmos o uso real dos termos supramencionados, extraímos as informações a respeito do contexto motivador destas categorias - por exemplo, bachelor não se aplica a um padre católico ou ao papa, apesar de ambos satisfazerem os requisitos para tanto: são homens solteiros que nunca se casaram ${ }^{2}$ - e pela análise da sua co-ocorrência colocacional em uso real - no caso de spinster, este é um substantivo comumente colocado com modificadores como elderly, gossipy, dried-up, dreary old, sex-starved, e repressed e verbos como remain. ${ }^{3}$ Em suma, estas breves análises nos forneceram vias para revelar o significado destas ULs em uso e nos mostram como o seu sentido revela que estas categorias não abrigam membros com as mesmas características no mundo real, mas que representam um protótipo culturalmente variável. Desta forma, pode-se dizer que os contextos em que bachelor é utilizado, bem como as colocações que encontramos para a palavra spinster em exemplos de uso real, constituem uma parte do frame destas ULs.

No último caso, isto quer dizer que, ao utilizar a palavra spinster, o falante invoca este frame, de forma que este conjunto de entendimentos se torna parte do seu discurso. Para fins de ilustração, podemos traçar uma comparação com o uso do adjetivo single. Ambas as ULs se subscrevem no frame geral Personal_relationship, ${ }^{4}$ mas poderão compor subframes ${ }^{5}$ distintos. Se dizemos "she is single" em vez de "she is a spinster", evocamos uma situação distinta, de certa forma mais neutra, pois, no primeiro caso, evoca-se um domínio conceitual povoado mais pela ideia de estado civil e elegibilidade do que de estagnação e incapacidade - uma é baseada na lei humana e costumes religiosos e sociais, a outra, na moral religiosa e de determinados grupos sociais. Assim, duas unidades lexicais que designam a mesma coisa (nos dois casos, fala-se de uma mulher não casada) situam o mesmo referente em frames distintos.

Este exemplo nos mostra como os frames cognitivos estão presentes no texto como o material lexical que evoca construtos relevantes na medida em que existe como indicador dos mesmos. Se o frame evocado consoante a escolha da UL identifica o cenário no qual se situa a proposição, os frames evocados no nível textual formam uma espécie de "visão do mundo" do texto, onde a sua compreensão depende da nossa capacidade de computar as informações subentendidas na construção de um contexto complexo no qual cada um dos frames sinalizados lexicalmente foi motivado (Fillmore 2006: 383).

Alguns autores descrevem o papel do framing lexical como o "conteúdo" que a gramática vem “configurar" (Fillmore 2006: 385; Langacker 2006; Talmy 2006), pelo que a gramática contribui com a estrutura esquemática básica para a organização conceitual no domínio cognitivo da linguagem. Um exemplo dessa interação é quando o frame de um verbo é configurado pelo tempo verbal empregado, de forma que esses elementos interagem formando uma história implícita subjacente à da narrativa. Por exemplo, em "she had been running", to run é configurado na narrativa

2 Do Oxford English Dictionary online: “A man who is not and has never been married”. Disponível em: https://www. lexico.com/en/definition/bachelor. Último acesso: 04/03/2021.

3 Exemplos extraídos de busca simples no British National Corpus, disponível em https://www.english-corpora.org/ bnc/. Último acesso: 04/03/2021.

4 Disponível para consulta na FrameNet pelo endereço https://framenet2.icsi.berkeley.edu/fnReports/data/ frameIndex.xml? frame=Personal_relationship. Último acesso: 05/05/2020.

Frames são grafados com letra maiúscula inicial e o símbolo de sublinhado (_) entre palavras, quando há mais de uma.

5 Quando um frame complexo é composto por outros frames, chama-se o primeiro super_frame e, os últimos, subframes (Ruppenhofer 2016: 9). 
pela forma do past perfect progressive, cujo papel é indicar que o estado atual da proposição é, em parte, explicado por um evento anterior ao tempo da narrativa. Assim, um enunciado como esse pode servir para explicar porque a personagem está sem fôlego, sendo que esta informação não se encontra no texto, mas no conhecimento do frame do evento "correr" (Fillmore 2006: 385).

\subsection{O projeto FrameNet}

Em termos concretos, um frame é um mapeamento entre significados conceituais complexos e dinâmicos e formas linguísticas. Refira-se o projeto FrameNet de Berkeley, ${ }^{6}$ que visa a compilação de frames da língua inglesa para consulta individual ou uso em aplicações de processamento de língua natural (PLN), onde as formas linguísticas assumem determinados papéis sintáticos. O referido projeto de semântica de frames "consiste en la creación de un lexicón de la lengua inglesa que contenga las posibilidades combinatorias semánticas y sintácticas (valencias) de cada unidad léxica" (Blanco-Carrión 2007: 73). Isto quer dizer que, ao decompor os frames e anotar frases em que as ULs ocorrem na língua em uso, a FrameNet identifica padrões de combinação semânticosintática, onde determinada aceção de uma palavra ou expressão é pareada às funções sintáticas que ela pode desempenhar.

Pela ferramenta de busca da FrameNet, acedemos a frames ou entradas lexicais formadas por uma ou mais palavras (ULs), listadas por frame, e um relatório de atributos de cada entrada lexical, entre outras informações. Se uma UL está listada em mais de um frame, há um relatório para cada frame ao qual pertence, que contém: o nome do frame, a definição simplificada da UL, os elementos do frame nos quais a UL se enquadra, exemplos de frases anotadas que contêm a UL (extraídos do British National Corpus) e as realizações sintáticas da UL nas frases anotadas.

Tomemos como exemplo novamente o adjetivo single, uma UL contemplada na FrameNet como duas entradas lexicais. A primeira inscreve-se no frame Personal_relationship e a segunda, em Sole_instance. Na primeira entrada, ${ }^{7}$ sua definição é "not involved in a stable romantic or sexual relationship", os elementos do frame aos quais se pode ligar são Partner 1 e Partner 2, cujas realizações sintáticas são descritas como N.Ext, N.Head, NP.Ext e INI, onde:

(i) N e NP referem-se a substantivo e sintagma nominal, respectivamente;

(ii) Ext (External): função gramatical que pode exercer a função de sujeito de verbos finitos e sujeito (ou objeto) de alguns verbos de controle ou direção;

(iii) Head: núcleo do sintagma;

(iv) INI (Indefinite Null Instantiation): um elemento de frame que não está realizado na frase e cuja identidade não se pode obter, mas cujo tipo normalmente é conhecido. ${ }^{8}$

6 A FrameNet é um projeto do International Computer Science Institute in Berkeley. Disponível para consulta pública em https://framenet.icsi.berkeley.edu/fndrupal/. Último acesso em: 02/07/2020. O projeto serviu como exemplo para a criação de outras framenets, como a espanhola, a alemã e a brasileira.

7 Disponível em https://framenet2.icsi.berkeley.edu/fnReports/data/lu/lu2044.xml?mode=lexentry. Último acesso em: 25/01/2020.

8 As definições de "Ext" e "INI" foram extraídas e traduzidas do glossário da FrameNet, disponível em: https:// framenet.icsi.berkeley.edu/fndrupal/glossary. Último acesso em: 25/01/2020. 
Por meio do mapeamento destas funções sintáticas (realizado a partir da anotação de frases retiradas do British National Corpus), são geradas valências, onde a combinação entre o sentido da UL e suas possíveis realizações sintáticas constituem evidências do tipo de estrutura em que as ULs podem ocorrer, consoante o sentido em que são empregadas na frase.

Quanto aos próprios frames, estes contêm, na FrameNet: uma descrição geral; os elementos de frame (EFs), com a sua descrição e exemplos de realização linguística; as relações que mantêm com outros frames da rede; e as ULs contidas na rede que se abrigam sob oframe. No caso de Personal_relationship, sua definição geral é:

The words in this frame have to do with people and the personal Relationships they are or can be a part of. Some of the words denote people engaged in a particular kind of Relationship, others denote the Relationship, yet others the events bringing about or ending the Relationships. [... $]^{9}$

Quanto aos elementos de frame, um EF é definido como "[a] frame-specific defined semantic role that is the basic unit of a frame". ${ }^{10}$ EFs são subdivididos em dois tipos: nucleares e não nucleares. Os elementos nucleares de Personal_relationship são Partner 1, Partner 2 e Partners, e os não nucleares, Degree, Depictive, Duration, Manner, Means, Relationship e Source of Relationship os primeiros são obrigatórios, pois são essenciais ao sentido do frame, ao passo que os últimos são opcionais, pois geralmente são “informações adicionais", como expressões temporais ou advérbios de lugar, modo etc. No exemplo acima, percebe-se que os Partners são elementos nucleares porque, sem os mesmos, não há relacionamento pessoal.

Assim, a FrameNet constitui um repositório para análise semântica que contribui para aplicações de PLN, para o aprendizado de línguas estrangeiras ou aprofundamento do conhecimento da língua materna, como defende Blanco-Carrión (2006), e para a tradução, pelo que fornece "estruturas conceituais refinadas" como fonte de pesquisa (Rojo \& Ibarretxe-Antuñano 2013: 21).

\subsection{Aplicação da semântica de frames à tradução}

Os exemplos mencionados nos permitem afirmar que, na abordagem da semântica de frames, a semântica lexical não pode ser descrita como um fenômeno isolado na língua, mas dependente da sua estrutura interna - onde se entrelaça às construções gramaticais na formação do sentido (Blanco-Carrión 2007) - e de uma estrutura conceitual de fundo partilhada pelos falantes. Desta forma, os frames constituem uma abordagem que circunscreve ULs relacionadas entre si a um pano de fundo mais abrangente que, sua por vez, relaciona-se com outros planos de fundo, as situações em que os falantes de uma língua podem se encontrar e as categorias nelas presentes. Contribuindo para a caracterização da motivação sociocultural presente nas línguas abrangidas neste estudo e a sua descrição linguística em termos formais, é nesta via

9 Disponível em: https://framenet2.icsi.berkeley.edu/fnReports/data/frameIndex.xml? frame=Personal_relationship. Último acesso em 26/01/2020.

10 Glossário da FrameNet, disponível em https://framenet.icsi.berkeley.edu/fndrupal/glossary. Último acesso: $14 / 09 / 2020$. 
que este trabalho se propôs a descobrir as categorias relacionadas à colocação single mother, revelando a estrutura conceitual prototípica da mesma na expressão de partida no inglês americano, na perspetiva da sua equivalência em português europeu (PE) e brasileiro (PB).

A incursão na tradução pelo prisma da semântica de frames já foi abordada por outros autores com objetivos diversos. Por um lado, Rojo (2002) aplicou a noção de frame à tradução pelo prisma cultural, propondo uma noção de equivalência tradutória orientada pela evocação dos mesmos frames na tradução (apud Rojo \& Ibarretxe-Antuñano 2013: 8). Nessa abordagem, o papel do tradutor seria guiar o leitor na construção e interação dos domínios conceituais presentes no texto como "a bilingual and bicultural 'mediator' between two different conceptual systems" (Rojo 2002: 315).

Bertoldi (2010 apud Boas 2013) desenvolveu um trabalho em terminologia jurídica comparada em inglês e português brasileiro valendo-se da semântica de frames em busca de termos equivalentes nos dois sistemas jurídicos e demonstrou diversos problemas de framing causados pela polissemia desses termos. Segundo Boas, a análise do autor demonstra

[...] that semantic frames are not only useful for comparing and contrasting translation equivalents, but that they are also helpful when it comes to highlighting differences in polysemy networks between languages and for showing how systematic cultural differences have direct repercussions for the organization of the conceptual system. (idem: 145)

Por outro lado, Kussmaul (1995) destacou a utilidade da semântica de frames para a compreensão e tradução de termos abstratos. Além disto, ilustrou como a noção de primeiro plano e plano de fundo - o profiling ou perfilhamento, que o autor subdivide em foregrounding e backgrounding - auxilia na tradução de termos complexos, pelo que permite identificar os planos mais relevantes ao contexto (apud Rojo \& Ibarretxe-Antuñano 2013: 8). O uso que fazemos da semântica de frames alinha-se à abordagem deste autor. Isto significa que o que entendemos por equivalência tradutória neste trabalho, diferente da abordagem de Rojo (2002) e Bertoldi (2010), alinha-se à dinamicidade do sentido linguístico encontrada entre as suas diferentes camadas textuais e conceituais.

\section{A virada cognitiva ${ }^{11}$ nos Estudos de Tradução}

Como mencionado, a Linguística Cognitiva constitui uma abordagem semântica ao estudo da língua que leva em consideração o ambiente cognitivo do falante, o seu conhecimento do mundo e a sua condição corporizada. Neste quadro teórico, a linguagem não é um módulo isolado da cognição humana, mas um reflexo da forma como o ser humano processa e armazena a informação - nesse sentido, é indissociável da experiência. Isto atribui caráter dinâmico ao significado, na sua interação com o mundo físico, social e cultural.

11 O termo virada cultural dos Estudos da Tradução é uma tradução nossa de cultural turn (Bassnett \& Lefevere 1990). Faber (2009) referiu o cognitive shift, que entendemos como a virada cognitiva. A autora usa o conceito aplicado à tradução de textos de especialidade e terminologia, enquanto o nosso objetivo ao nos apropriamos do mesmo é nos referirmos à tradução de modo geral. 
Para a tradução, esta abordagem implica um retorno investigativo ao símbolo linguístico com vistas a revelar essa estrutura conceitual subjacente no processo de interpretação e "mapeá-la" no texto de chegada, sem, contudo, deixar de considerar as restrições da forma linguística e os aspetos pragmáticos relacionados ao seu uso em ambientes cognitivos distintos. Desta forma, procuramos situar o signo linguístico no quadro da Linguística Cognitiva, a fim de circunscrever o que se pode entender por equivalência tradutória no contexto desta abordagem.

\subsection{A natureza do sentido linguístico}

A visão da unidade de sentido invariante liga-se ao paradigma clássico que descreve a cognição humana como a manipulação de representações simbólicas, um processo que segue as regras da linguagem mental (por exemplo, Fodor 1983 apud Martín de León 2013: 100). Nesse paradigma, segundo Martín de León (idem), a linguagem mental é independente da língua natural, que é vista como um instrumento de comunicação, mas não de pensamento. O sentido, nesta perspetiva, surgiria a partir da combinação sintática dessas representações simbólicas (Glenberg \& Roberston 2000 apud Martín de León 2013: 100), que seriam arbitrárias e amodais:

[...] arbitrary because there is no formal relationship between them and their referents, and (...) amodal because they are independent of sensorial modalities, i.e. because there is no correspondence between their structures and the perceptual states that caused them to exist. (Barsalou 1999 apud Martín de León 2013: 100)

Por outras palavras, símbolos arbitrários não podem ter significado porque não há um construto que os relacione a entidades reais no mundo - é isto que postula o chamado symbol-grounding problem (Harnad 1987, 1990 apud Martín de León 2013: 101). Além disto, símbolos amodais não podem ter significado sem uma relação direta com a experiência percetiva, motora e emocional (De Vega 1984 apud Martín de León 2013: 102). Contudo, o ser humano - que adquire a linguagem em contexto comunicativo - não tem dificuldade em mapear esses símbolos aos seus referentes no mundo real, o que leva a autora a concluir que o significado atribuído a sistemas de símbolos abstratos depende necessariamente do intérprete externo (Martín de León 2013: 101).

Por outro lado, quando a construção do sentido é vista como um processo incorporado, complexo, dinâmico e situado, o significado deixa de ser inerente ao sistema simbólico e passa a depender da cognição individual e dos sentidos partilhados no seio de comunidades linguísticas, de forma que a correspondência interlínguística na tradução não pode ser absoluta. Além disto, o facto de o sentido se afigurar dinâmico e incorporado é atribuível às dimensões da experiência, representadas na mente humana:

[...] do ponto de vista da Linguística Cognitiva, o acto de tradução reporta-se à ativação e ao uso seletivo de estruturas de conhecimento, filtradas pela mente do tradutor. Portanto, a questão central das equivalências em tradução está para além da dimensão linguística propriamente dita, envolvendo antes um conjunto complexo de ligações e associações na mente do tradutor. (Almeida 2016: 2) 
Naturalmente, a ideia da impossibilidade de se alcançar a equivalência total entre texto de partida e texto de chegada não é nova na teoria da tradução. O que se postula, pelo contrário, é que conceber o sentido como dinâmico - em sua camada formal, denotativa, conotativa, pragmática ou funcional (Martín de León 2013: 108) - nos fornece caminhos para acedê-lo e pode informar o processo de eleição das prioridades da tradução consoante a situação de comunicação. Nesse sentido, a tarefa do tradutor aparece não como manipulação de símbolos arbitrários entre línguas, mas como aquela que fornece aos leitores as ferramentas de que precisam para construir o seu próprio sentido em sua própria situação (Risku 2004 apud Martín de León 2013: 99). Além disto, entender as operações de construção do sentido como parte integrante do processo de tradução nos permite manter muitas das ferramentas e metodologias dos Estudos de Tradução na prática (Halverson 2013: 48).

\subsection{O sentido dinâmico aplicado à tradução}

A premissa da Linguística Cognitiva de que a língua natural é parte integrante da cognição sustenta a ideia de que o processo de tradução envolve mais do que a transposição de estruturas linguísticas, sendo que postula a natureza cognitiva da tradução enquanto mediação entre mundos conceituais distintos. Assim, aplicar a noção de sentido construído conceitualmente à tradução - que há muito procura lidar com a sua instabilidade - nos permite integrar o conhecimento linguístico a influências sociais, históricas e conceituais por meio do processo cognitivo do tradutor (Rojo \& Ibarretxe-Antuñano 2013: 19).

O presente trabalho descreve o processo de tradução da colocação single mother, uma expressão composicional da linguagem comum, não especializada. Segundo a definição de unidades terminológicas de Faber (2009) que apresentamos abaixo, a tradução de terminologia muito tem em comum com a tradução de colocações da linguagem não especializada - além de possuírem significados específicos em contexto e valência sintática, a colocação em questão é um sintagma nominal, o que facilita ainda mais o aproveitamento da definição da autora. Segundo Araúz, Faber \& Martinez (2012), as unidades terminológicas são

[...] mostly represented by compound nominal forms that are used within a scientific or technical field, and have meanings specific of this field as well as syntactic valence or combinatory value. (idem, p. 120)

Assim sendo, estas autoras procuram fazer a ponte entre a tradução de terminologia e textos de especialidade e a Linguística Cognitiva, argumentando que o sentido lexical não deixa de ser dinâmico na linguagem de especialidade, apesar da visão tradicional do campo da Terminologia de que unidades terminológicas tendem a ter referentes mais específicos e a ser menos polivalentes (2009: 111). Além disto, como as unidades terminológicas são carregadas de sentido e se inserem em uma estrutura conceitual que está raramente presente no texto, Wright \& Wright (1997 apud Faber \& Gómez-Moreno 2012: 83) afirmam que o tradutor frequentemente se encontra na posição de terminológo ad hoc, que precisa reconstruir partes de sistemas conceituais em vez de estruturar áreas de conhecimento especializadas. Sendo assim, a análise terminológica não escapa 
à necessidade de conhecimento da estrutura conceitual subjacente à forma linguística, além de apresentar padrões sintáticos e de combinação específicos. Assim sendo, Faber \& Goméz-Moreno (2012) aplicam a sua abordagem à terminologia a uma teoria da tradução de linguagem de especialidade com base na semântica de frames, em cujos construtos se apoia para aceder o sentido das unidades terminológicas e estruturar os conceitos ancorados a domínios conceituais específicos.

Esta abordagem tem aplicação relevante no presente trabalho não só por elucidar o nosso recurso à semântica de frames como porta de entrada ao significado das unidades lexicais que traduzimos, mas também porque traz à tona a noção de plano de fundo e perfilhamento (profiling) central na LC. A ideia de Langacker (2006: 31-32) de que o sentido linguístico é construído a partir de relações entre base e perfil, onde o significado de determinada unidade lexical se dá por referência a um domínio mais amplo - por exemplo, a palavra ponta (o perfil) é entendida por referência à sua base (a lâmina de uma faca, a extensão de um bastão etc.) também é fundamental na semântica de frames, que postula que o significado lexical só pode ser definido por referência à estrutura conceitual na qual se insere (Fillmore, 2006: 373), como exemplificado anteriormente. Pretende-se, portanto, que os frames sejam uma representação dessa estrutura, um quadro que mapeia a relação entre unidades lexicais e a estrutura conceitual subjacente que as abriga. Nessa abordagem, não se pode fazer uma distinção clara entre semântica e pragmática, visto que (i) o significado linguístico estaria intimamente ligado ao ambiente cognitivo do falante, da comunidade linguística e dos seus textos e (ii) a priori pode-se ter acesso, por exemplo, às pressuposições e implicaturas do discurso, por recurso aos frames nos quais as unidades lexicais se enquadram.

Segundo Rojo \& Ibarretxe-Antuñano (2013: 21), a semântica de frames permite aceder ao processo de construção do sentido em regime de tradução, na medida em que se baseia na análise dos campos semânticos intra- e interlinguisticamente. Ou seja, a semântica de frames constitui uma metodologia de análise semântica que permite salientar semelhanças e diferenças entre diferentes línguas. Ao orientamos a nossa análise da colocação single mother pela via pragmático-conceitual, especificamente por recurso à semântica de frames, o nosso olhar tradutório não deixa de considerar, como não poderia, a dimensão linguística e comunicativa das unidades lexicais. Alinhada à semântica de frames e à chamada "semântica da compreensão" de Fillmore, a nossa escolha de perfilhamento - a dimensão mais saliente da unidade lexical - é regida pela sua relevância contextual. Ou seja, levamos em consideração o conhecimento subjacente e a relevância do mesmo para o contexto, a sua forma linguística e o tipo e propósito da situação de comunicação.

Contudo, a relevância contextual nem sempre é acessível à primeira vista. Também alinhada aos postulados da Linguística Cognitiva, o princípio da relevância de Ernst-August Gutt fornece mais bases para guiar as escolhas do tradutor no perfilhamento entre as dimensões da semântica linguística, no sentido exemplificado no trabalho de Kussmaul (1995) supramencionado. Gutt defende que transmitir a "mesma mensagem" do texto de partida no texto de chegada inclui transmitir tanto as implicaturas quanto as explicaturas. Assim, "a translation would be a receptor language text that interpretively resembled the original" (2000: 376, grifo nosso).

Put more generally, in interpretive use the principle of relevance comes across as a presumption of optimal resemblance: what the reporter intends to convey is (a) presumed to interpretively resemble the original $[\ldots]$ and (b) the resemblance it shows is to be consistent with the presumption of 
optimal relevance, i.e. is presumed to have adequate contextual effects without gratuitous processing effort. (idem: 377 , grifos nossos)

Quanto à relevância, o autor põe em destaque tanto os efeitos contextuais - "modificações na memória de longo prazo [...] que decorrem basicamente da conjunção de elementos resultantes do processamento linguístico com certos conhecimentos prévios [do] receptor" (Pagano et al., 2005: 65) - quanto a importância de se observar o "esforço de processamento" que o texto de chegada exige do leitor. Na Linguística Cognitiva, as noções de nível básico de categorização e o uso que faz da Teoria dos Protótipos (Rosch 1973, 1975 e 1978), além da noção de perfilhamento supra, se alinham à ideia de mínimo esforço de processamento, pelo que representam construtos de "atalhos cognitivos" na construção do sentido linguístico. Aprofundando esse ponto com foco na tradução, Gutt (2000) discorre sobre o caminho interpretativo do leitor, a fim de demonstrar como o princípio da relevância orienta a tradução, quer em termos de conteúdo, quer em termos de forma.

Como já apontou Steiner (2000: 186), o leitor deposita no texto a confiança de que há um sentido a se extrair e se implica no processo de interpretação. Segundo Gutt e alinhado à visão de Fillmore (1982/2006) sobre o papel dos frames, no processo de construção do sentido procura-se, por ordem de relevância, identificar as razões pelas quais uma determinada forma linguística foi escolhida para exprimir determinada ideia:

[...] it is not simply the first interpretation that comes to mind that the audience is entitled to take as the intended interpretation, but rather the first interpretation that comes to mind and that is consistent with the principle of relevance. (2000: 380, grifo nosso)

Para exemplificar esse ponto, o autor cita o comentário de Adams (1973: 14) a uma passagem da tradução para inglês de C.K. Scott-Moncrief do romance Le rouge et le noir, de Stendhal. Em determinada passagem, o tradutor utiliza a forma pronominal thou como tradução para o francês $t u$ - presumidamente no intuito de preservar a relação de intimidade que esta forma implica - em detrimento do pronome you, mais moderno e neutro em termos de registro. A cena do romance deveria sinalizar que um rapaz havia "caído novamente nas graças" da mocinha, visto que essa se lhe dirigiu por $t u$. Entretanto, Gutt acredita que a escolha seja infeliz porque, ao procurar manter esta implicatura na tradução - manter a semelhança de interpretação -, o tradutor teria errado ao utilizar uma forma menos acessível ao leitor em termos de esforço de processamento e efeitos contextuais adequados, pois o difícil caminho para alcançar esse sentido, que não está disponível para a "maioria das pessoas" (considerando-se o anacronismo da forma thou), não corresponde ao caminho interpretativo do $t u$ presente no texto de partida (Gutt 2000: 378-380).

Com esse e outros exemplos, como as situações de tradução simultânea - onde reduzir o esforço de processamento é ainda mais relevante do que pormenores da mensagem e da forma linguística - e tradução para teatro, onde considerações inerentes ao efeito dramatúrgico e à forma de apresentação da mensagem frequentemente se sobressaem, Gutt afirma que a tradução como uso interpretativo interlinguístico com base no princípio da relevância soluciona a busca por um conceito de fidelidade tradutória que é ao mesmo tempo generalizável e alinhado à especificidade do texto e do contexto: 
It is generally applicable because it involves only notions believed to be part of human psychology - the principle of relevance and the ability to engage in interpretive use. It is text-specific in that interpretive use will link the communicative intention of the translator to the intended interpretation of the original text. It is context-specific in that the search for consistency with the principle of relevance always brings in the particular cognitive environment of the audience addressed. (2000: 393)

Torna-se claro, portanto, que a proposta de Gutt está alinhada aos construtos da Linguística Cognitiva, pelo que contempla a construção do sentido quer no aspeto cognitivo, quer linguístico, quer também pragmático. Desta forma, aplicamos ao nosso processo de tradução a noção de uso interpretativo por recurso à semântica de frames no intuito de exercitar uma noção de equivalência tradutória alinhada à dinamicidade do sentido e à interação entre as suas várias dimensões, em consonância com esses postulados.

\section{Contextualização da situação de comunicação de single mother: um corpus sobre a liderança no feminino}

O acervo de colocações no qual single mother se insere, cuja equivalência em tradução inglês-português será o foco deste estudo, integra o frame Female_leadership. Este foi compilado mediante extração de colocações de um corpus que versa a liderança no feminino, composto pelas publicações Women Don't Ask - Women and the Gender Divide, A Woman's Guide to Sucessful Negotiation e Lean In - Women, Work and the Will to Lead (Babcock \& Laschever 2003; Miller \& Miller 2011; Sandberg \& Scovell 2013, respetivamente). De modo geral, os textos do corpus procuram descrever o estado das coisas no que concerne à posição da mulher na sociedade, às relações de trabalho e, em menor medida, ao poder político. Nestas três obras são abordadas questões sociológicas e psicológicas que podem ajudar o público leitor a compreender as razões subjacentes à atual situação da mulher na sociedade, gizando o caminho a percorrer para conseguir uma maior igualdade entre os gêneros.

No âmbito acadêmico, os Estudos de Gênero e estudos em Sociologia e Psicologia das últimas três ou quatro décadas têm procurado identificar as razões pelas quais as mulheres, apesar do progresso na conquista de igualdade de oportunidades e direitos, ainda ocupam, em muitos países, fundamentalmente posições de liderança na esfera do lar, em detrimento de funções de liderança na esfera política ou do mercado de trabalho. O corpus em questão (Babcock \& Laschever 2003; Miller \& Miller 2011; Sandberg \& Scovell 2013) se utiliza desses estudos para interpelar as mulheres profissionalmente ativas. Além de elencar os problemas estruturais que as mulheres enfrentam, os textos abordam formas de consciencialização das vantagens e desvantagens do perfil psicológico e social inerentes ao feminino. Ou seja, por um lado, os textos do corpus ilustram, pelo prisma das ciências humanas, a estrutura social que constrói e perpetua papéis supostamente adequados aos gêneros [gender roles] ${ }^{12}$ e os estereótipos a eles relacionados [gender stereotypes].

12 Os termos entre colchetes [square brackets] nesta seção indicam a colocação em inglês (originária do corpus) ou português (tradução nossa) correspondente àquela mencionada no corpo deste texto e que compõe a base de dados completa de colocações bilingue que desenvolvemos na dissertação do Mestrado em Tradução na Faculdade de Letras da 
Por outro, sublinham o facto de o comportamento feminino ser condicionado por estes papéis sociais. Nesta linha, se envereda quer pelo questionamento e desmantelamento da estrutura social que restringe o papel da mulher na sociedade e no mundo do trabalho, quer pela apresentação de formas de ação para que a própria mulher possa reverter a sua situação no mundo, mediante consciencialização do valor das valências humanas e sociais do feminino na sociedade.

No plano estrutural, argumenta-se, com base em estatísticas, que a diferença entre os gêneros [gender difference] compreende não apenas o espaço que a mulher ocupa no mercado de trabalho e na política, mas, para citar alguns exemplos, a sua remuneração e condições de trabalho em diversos níveis, fundamentalmente no que respeita a diversas formas de dualidade de critérios [double standards] de contratação, promoção e atribuição de tarefas. No substrato argumentativo da exposição deste retrato, encontramos nos três livros do corpus de trabalho colocações como gender gap, gender bias, market value (of women), true equality, hiring criterion, labor force participation, entre outras.

Diversos estudos (por exemplo, Thompson 1998 apud Babcock \& Laschever 2003: 180) também demonstraram que, além da vida profissional, a mulher casada [married woman] assume muito mais tarefas domésticas [housework]. No seu papel de mãe, também assume mais responsabilidades na criação dos filhos [child rearing; child care], o que faz com que as mulheres desfrutem de menos momentos de lazer [leisure time]. Assim sendo, encontramos uma amostra significativa das responsabilidades familiares da mulher casada e/ou mãe nas colocações single mother, good mother, woman with children, employed mother, stay-at-home mother, entre outras. Estas e outras colocações, como good listener e child care, formam um subframe de Female_leadership que chamamos Papéis_sociais.

\subsection{Metodologia de levantamento das colocações do corpus}

Como mencionado, single mother é uma das colocações extraídas do corpus selecionado sobre liderança no feminino. A lista de colocações completa, compilada para análise sob o prisma da semântica de frames e posterior tradução, contém 80 colocações de duas ou mais palavras extraídas do corpus de trabalho completo (256.219 palavras no total) por recurso ao conjunto de ferramentas Sketch Engine (Lexical Computing Ltd.). O serviço online do Sketch Engine (SkE) possibilita a extração de palavras ou colocações com foco na frequência de ocorrência das mesmas em determinado corpus ou em comparação a um corpus de referência que serve como contraponto de linguagem geral em comparação à linguagem especializada do corpus selecionado. Para este efeito, utilizamos o English Web 2015 (enTenTen15), composto por textos de conteúdo geral extraídos da internet e processados de forma a excluir os dados não relevantes, duplicações, spams etc. ${ }^{13}$ Este corpus de referência pertencente à família TenTen é anotado pelo sistema TreeTagger com uso das etiquetas do projeto Penn TreeBank (University of Pennsylvania), com algumas modificações (Marcus et al. 1993).

Universidade de Lisboa: A liderança no feminino em tradução: uma perspectiva cognitiva centrada em frames, escrita por Marilia Sette Câmara (2021) e orientada pelas Professoras Doutoras Maria Clotilde Almeida (Universidade de Lisboa) e Olga Blanco-Carrión (Universidade de Córdoba).

13 Encontra-se uma descrição completa do processo de preparação dos corpora extraídos da internet utilizados pelo SkE em https://www.sketchengine.eu/build-a-corpus-from-the-web/. 


\subsubsection{Seleção qualitativa preliminar das colocações}

A primeira etapa de seleção qualitativa da lista de colocações dentre as 231 colocações iniciais resultou na exclusão de 86 colocações imediatamente identificadas como não relevantes, restando 145 colocações para a próxima etapa de análise. Entre as colocações excluídas, encontraram-se: locuções conjuntivas (ex. (in the) first place); referências bibliográficas; repetições (ex. low sense e sense of entitlement, quando a colocação é low sense of entitlement); expressões muito comuns encontradas em uma grande variedade de contextos (ex. good idea e big difference); expressões relacionadas ao gênero textual do corpus (ex. next chapter), entre outras categorias.

A segunda etapa de análise qualitativa das 145 colocações restantes foi iniciada com a elaboração de um documento do Word (Microsoft Office 2013) contendo todas as colocações e respetivas concordances, extraídas manualmente mediante recurso à ferramenta Concordances do SkE. A esta lista de concordances, ainda se acrescentou outras colocações extraídas manualmente por meio da ferramenta Word Sketch do SkE juntamente com suas concordances. A ferramenta Word Sketch foi utilizada com o propósito de eliminar a possibilidade de deixar colocações relevantes sem identificação, bem como de obter o máximo de colocações relevantes possível, por forma a obter uma amostra lexical suficientemente representativa do frame Female_leadership. Além de a ferramenta Keywords calcular os resultados obrigatoriamente por comparação a outro corpus, cuja fórmula de cálculo do score pode levar à exclusão de algumas colocações importantes para esta pesquisa, ela também não lista as colocações por lema, mas sim por vocábulo. Registe-se que o lema difere do vocábulo porque inclui todas as variações gramaticais do mesmo, como variações de gênero e número, ou entre adjetivos comuns, comparativos e superlativos. Ademais, a funcionalidade Keywords também desconsidera expressões hifenizadas, podendo assim ocultar mais resultados relevantes.

A ferramenta Word Sketch processa as colocações a partir do lema, apresentando os collocates em diversas listas, por categorias gramaticais. Exemplos destas categorias são nouns modified by $X$, modifiers of $X$, verbs with $X$ as subject (onde " $X$ " é o lema buscado), entre outras. A nossa busca considerou as categorias que envolvem substantivos e modificadores, ${ }^{14}$ considerando-se que procuramos encontrar as colocações relevantes para o nosso frame excluindo verbos e sintagmas verbais (que iriam requerer abordagens metodológicas diferentes). Em seu conjunto, os resultados da busca com a Word Sketch apresentam "um resumo do comportamento colocacional e gramatical da palavra". 15

As palavras incluídas nas buscas com a Word Sketch foram selecionadas: (i) aleatoriamente (leader, woman, power, level, gender, entre outras); (ii) a partir da leitura dos textos do corpus, quando notamos expressões frequentes ou que consideramos importantes para o contexto da pesquisa (stereotype, satisfaction, barrier, family, wages, social, entre outras); (iii) a partir da lei-

14 Neste trabalho, consideramos como modificadores substantivos, adjetivos e locuções adjetivas que modificam substantivos em inglês; na tradução para as variantes do português, tornam-se adjetivos ou locuções adjetivas (grupos adjetivais ou preposicionais - por exemplo, low sense of entitlement, uma colocação que ocorre no trabalho referido de Câmara (2021) é traduzida por sentimento de inferioridade).

15 Traduzido da descrição da ferramenta disponível em https://app.sketchengine. $\mathrm{eu} / \#$ wordsketch?corpname=user\%2Fmariliasette\%2Ffemale_leadership\&tab=about\&lemma=female\&showFreqs=1\&sort_ ws_columns=f. Último acesso em: 10/03/2020. 
tura dos textos do corpus em busca especificamente de itens hifenizados, que normalmente não aparecem nas buscas automáticas do SkE (stay-at-home, male-dominated, decision-making/maker, entre outras); (iv) a partir da lista de single-word keywords gerada pela ferramenta Keywords, que nos fornece as palavras isoladas mais recorrentes no corpus de trabalho. As buscas resultaram no acréscimo de 58 colocações à lista inicial em formato Word, às quais também acrescentamos as respetivas concordances. Portanto, a lista final das colocações selecionadas até este ponto e com as respetivas concordances em formato Word reunidas para análise qualitativa e enquadramento em frames contempla 203 colocações.

\subsubsection{Seleção quantitativa e qualitativa manual das colocações em contexto}

Procedemos à análise da lista de 203 colocações em formato Word analisando as concordances das colocações, uma por uma, e excluindo aquelas que não correspondiam ao mesmo contexto (ou seja, que não fossem ocorrências das mesmas ULs) ou que não se encontravam no texto propriamente dito, mas nos paratextos: índices e referências bibliográficas, por exemplo. Como mencionado anteriormente, as colocações que somavam menos de cinco concordances relevantes foram excluídas da análise.

Diversas colocações também foram excluídas nesta fase, por terem sido julgadas irrelevantes ao frame Female_leadership. Muitas delas são unidades lexicais do campo da negociação e cujo significado se encontrava acima da divisão de gêneros - ou seja, não dizem respeito especificamente à mulher, ao homem, e tampouco suscitam diferenças entre gêneros. Alguns exemplos são: other side/party/negotiatior e reasonable offer; negotiating paradigm e negotiating style, entendidos como hiperónimos de estilos específicos, como relational e competitive style, que foram incluídos por serem associados à preferência por um ou outro gênero; bottom line - especificamente no contexto de negociação, esta UL designa a condição mínima aceitável para que as partes cheguem a um acordo; asking price e total compensation, citados como parâmetros para a negociação de salários; good deal e give-and-take, de entre outras relacionadas ao processo de negociação; social cue, no contexto de negociações virtuais; e body language, cujas ocorrências não abordam especificamente questões relacionadas aos gêneros. Algumas expressões foram excluídas por seu caráter geral, também acima da divisão de gêneros - entre elas, encontram-se different approach, prospective employer e personal style.

Em contraste às colocações excluídas por seu caráter demasiado geral, também excluímos colocações utilizadas em contextos demasiado específicos. Entre elas, destacam-se long-term dream, TV station manager e um conjunto de colocações que evoca o frame do divórcio. Ao final desta etapa, de 203 colocações restaram 80 que compuseram a lista de colocações em inglês posteriormente traduzida para português europeu e brasileiro. 


\section{Framing das colocações em Female_leadership}

As 80 colocações extraídas do corpus foram enquadradas em 31 frames. Os frames mais representados nas colocações foram: Gender_issues [Questões_de_gênero], Capability [Capacidade_ação], Societal_roles ${ }^{\star 16}$ [Papéis_sociais ${ }^{\star}$ ], Leadership [Liderança], Similarity [Igualdade\#], Kinship [Parentesco], Partiality [Parcialidade], Organizational_hierarchy ${ }^{\star}$ [Hierarquia_organizacional $^{\star}$ ], Being_employed [Ser_empregado] e Work [Trabalhar]. Outros frames evocados foram Hiring [Contratar], Success_or_failure [Sucesso_ou_fracasso] e Personal_relationship [Relações_ pessoais]. Note-se que algumas colocações composicionais podem se enquadrar em mais de um frame, visto que núcleo e modificador podem evocar frames diferentes do sentido veiculado na colocação. Logo, se considera que o substantivo nuclear, quando é modificado por substantivos ou adjetivos que não evocam subframes de Female_leadership, podem integrar frames distintos. Este é o caso da colocação single mother, que se enquadra em três frames distintos, a saber: Kinship, Personal_relationship e Societal_roles*.

Em Kinship ${ }^{17}$ - um frame que contém palavras que denotam relações de parentesco -, nesta e em outras colocações, o substantivo mother ocorre com modificadores que determinam a relação da mãe com o trabalho - como o caso de stay-at-home mother, que se refere a mães que não trabalham fora de casa, e single mother, que se refere a profissionais com filhos e cabeças de famílias monoparentais - ou evocam a dualidade de papéis da mãe que trabalha fora, o caso de good mother. Esta caracterização, da perspetiva da semântica de frames, nos diz, por exemplo, que o fato de uma mulher ser ou não casada e ter ou não filhos é relevante para a sua situação profissional e status social, considerando-se que oframe Kinship está aqui representado como subframe de Female_leadership.

O frame Personal_relationship ${ }^{18}$ inclui ULs que designam relações pessoais, as pessoas nestas relações e os eventos que dão início ou fim às relações (por exemplo, casamento e divórcio). Como subframe de Female_leadership e segundo o corpus, duas colocações se enquadram nele: single mother e married woman. Isso indica que, nos contextos do corpus, o estado civil da mulher, além do seu papel de mãe, está relacionado de alguma forma à sua representatividade em posições de liderança.

As duas obras Women Don't Ask e Lean In (Babcock \& Laschever 2003; Sandberg \& Scovell 2013), que integram o corpus deste trabalho, abordam a situação da mulher na sociedade, em casa e no trabalho. Logo, a partir destes enquadramentos sociais, observa-se alguns papéis que a mulher assume ou que se espera que assuma no contexto social. São, em última instância, responsabilidades que lhe são socialmente atribuídas e que estão ligadas aos padrões de gênero e comportamento social referidos na seção anterior: a mulher é comunal (valoriza o bem-estar comum mais do que o seu próprio), ela cuida, ela é mãe. Para abrigar essa situação, criamos o frame

16 Legenda: Os frames sinalizados com asterisco $\left(^{*}\right)$ não constam na FrameNet de Berkeley e nem na FrameNet Brasil, tendo sido criado pelas autoras; os frames sinalizados com hashtag (\#) constam na FrameNet de Berkeley, mas não se encontram traduzidos na FrameNet Brasil, de onde extraímos os frames que utilizamos em português.

17 Disponível na FrameNet de Berkeley em https://framenet2.icsi.berkeley.edu/fnReports/data/frameIndex. xml?frame=Kinship. Último acesso: 04/03/2021.

18 Disponível na FrameNet de Berkeley em https://framenet2.icsi.berkeley.edu/fnReports/data/frameIndex. xml?frame=Personal_relationship. Último acesso: 04/03/2021. 
Societal_roles a partir do mapeamento metafórico daquele existente na FrameNet de Berkeley como Performers_and_roles. ${ }^{19}$ Este frame compreende as ULs que evocam a situação em que um Intérprete tem determinado papel em uma performance e o interpreta segundo as orientações do roteiro para aquele papel, neste caso aplicado às interações sociais.

Incluímos em Societal_roles colocações que funcionam como as target words ${ }^{20}$ do frame, como role, presente em gender role e woman in leadership role. Os rótulos dos papéis sociais incluem single mother, stay-at-home mother, good mother, woman with children e employed mother - o fato de uma mãe ser boa ou não é um papel social atribuído a ela, assim como a mãe que fica em casa ou trabalha fora e a mãe a solo/mãe solo.

\section{De single mother a mãe a solo ou mãe solo}

Nos últimos anos, o uso da colocação mãe solteira, a tradução mais consagrada de single mother, tem sido questionado no ambiente social, especialmente nas interações pela internet, em diversos lugares no mundo, inclusive em Portugal, no Brasil, nos EUA - onde também se utiliza solo mother - e em França, onde maman solo tem ganhado terreno, em detrimento de mère célibataire. Na designação comum e enquanto expressão fixa, a mãe solteira não é casada e assume toda ou a maior parcela de responsabilidade na criação do(as) filhos(as). Contudo, uma pessoa não ser casada não só não quer dizer que seja solteira (pode estar em algum relacionamento, ser divorciada ou viúva, por exemplo), como, principalmente, não se acredita que o seu estado civil seja a circunstância mais relevante. Pelo contrário, preconiza-se que o seu modus vivendi seja caracterizado pelo fato de cuidar do(as) filho(as) majoritariamente sozinha - é ela quem coabita com a(s) criança(s), de forma que toda ou a maior parte do trabalho de criação, educação e cuidado recai sobre ela mesma. Por esse motivo, nos últimos anos temos observado o uso da expressão mãe a solo/mãe solo em português europeu (PE) e mãe solo em português brasileiro (PB).

Segundo Borges (2020: 1-2), o termo mãe solteira carrega o resquício da sociedade patriarcal do século $\mathrm{XX}$, onde os direitos civis, sexuais e reprodutivos da mulher casada eram reduzidos e, em grande parte, submetidos à vontade do marido. Além disso, o casamento era considerado como a única possibilidade de constituição de família. Segundo a autora, esta situação estaria relacionada à moral que veio se instituir no Brasil com o Código Civil de 1916,

[...] que previa dentre outros dispositivos a exigência da virgindade da mulher no momento do casamento, obrigatoriedade da mulher de acrescentar ao seu nome o sobrenome do marido, proibia a investigação de maternidade cuja prole decorresse de relação havida fora do casamento, extinguia o poder familiar das mães sobre os(as) filhos(as) havidos em relacionamento anterior, caso esta contraísse novas núpcias e incluía as mulheres casadas no rol de relativamente incapazes, precisando de autorização do marido para herdar, trabalhar, alienar imóvel, aceitar mandato, litigar em juízo cível ou comercial, dentre outras limitações aos atos da vida civil. (idem: 4)

19 Disponível na FrameNet em https://framenet2.icsi.berkeley.edu/fnReports/data/frameIndex.xml?frame=Performers_ and_roles. Último acesso em 27/05/2020.

20 Target words são ULs que evocam determinado frame independentemente de contexto (Ruppenhofer et al., 2016, p. 20) 
Sublinhe-se que a colocação mãe solteira enquadra-se nos frames Relações_pessoais (evocado por solteira), Parentesco (mãe) e Papéis_sociais (mãe solteira), da mesma forma que single mother enquadra-se em Personal_relationship (single), Kinship (mother) e Societal_roles (single mother), conforme descrito anteriormente.

Borges (2020: 2) também cita instrumentos jurídicos brasileiros, como o Estatuto da Mulher Casada (Lei no 4.121/1962), a Constituição Federal de 1988, o Estatuto da Criança e do Adolescente (Lei $\mathrm{n}^{\circ}$ 8.069/90) e o Código Civil de 2002, entre outros, não só como essenciais para a emancipação da mulher, mas também para o alargamento do conceito de família para além da relação conjugal. Além disto, observa-se de fato tanto em Portugal quanto no Brasil um aumento expressivo de famílias monoparentais lideradas por mulheres - em 2010, Portugal registava 300.677 famílias lideradas por mães a solo, ${ }^{21}$ o que representa $86.8 \%$ das famílias monoparentais do país; no Brasil, este número chegava a 9,14 milhões de famílias no mesmo ano e $42 \%$ dos domicílios comandados por mulheres têm chefes de família a solo. ${ }^{22}$ Em vista desta transformação na sociedade, observa-se a transformação na linguagem e a adoção cada vez mais ampla do termo mãe a solo/mãe solo. Segundo Silva et al. (2019: 2),

[...] a expressão mãe solo tem se popularizado na sociedade atual como uma tentativa de desconstruir a definição pejorativa e relacionada ao estado civil. Por muito tempo, o termo foi tratado sob a visão do controle social nas sociedades patriarcais, na qual a maternidade se apresenta como elemento de subjugação da mulher em relação ao homem. Mudar a forma de se referir a essas mulheres visa, desta maneira, eliminar o preconceito com as genitoras que não têm qualquer relação com o pai de seus filhos, ou com mães que se separam, ou mães que optaram por serem mães sem necessariamente estar em uma relação conjugal.

Em PB, o modificador apositivo solo é utilizado com valor adjetival no sentido de "realizado, desempenhado, tocado, executado etc. por uma pessoa apenas" e, apesar de a locução a solo se encontrar lexicalizada com o mesmo significado, ${ }^{23}$ a primeira forma é mais corrente na língua brasileira atual - ao que tudo indica, por influência do uso de solo como modificador em inglês, que possui valor adjetivo e advérbio lexicalizado. ${ }^{24} \mathrm{Em} \mathrm{PE}$, é possível encontrar tanto a forma a solo quanto o apositivo solo em uso e lexicalizados. ${ }^{25}$ Embora a primeira seja mais utilizada de modo geral no PE falado, e considerada mais feliz pelos falantes nativos, observa-se o uso esporádico de mãe solo ${ }^{26} \mathrm{o}$ que supomos estar relacionado à maior quantidade de ocorrências da expressão

21 Fonte: https://www.pordata.pt/Portugal/Agregados+dom\%c3\%a9sticos+privados+monoparentais+total+e+por+s exo-20 citado em Baliana (2013).

22 Fontes: Instituto Brasileiro de Geografia e Estatística (IBGE) e Secretaria de Políticas para as Mulheres (SPM) (BRASIL, 2019) citado em Silva et al. (2019).

23 Do Dicionário Antônio Houaiss, exemplos: "violino solo", "voo solo", "ginástica a solo".

24 Do Oxford English Dictionary online: "Adjective: Done by one person alone; unaccompanied; Adverb: For or by one person alone." (https://www.lexico.com/en/definition/solo)

25 Do Dicionário Priberam: “"solo”, adjetivo de dois géneros e dois números: (i) Que tem apenas um executante, (ii) Que se apresenta desacompanhado; "a solo", (i) Que é executado por um único indivíduo, (ii) Que está sozinho = desacompanhado."

26 Ver, por exemplo, "Pais e mães a solo", em https://ifestyle.sapo.pt/familia/pais-e-filhos/artigos/pais-e-maes-a-solo. Último acesso: 04/03/2021. 
em PB em publicações nos média online e nas redes sociais digitais, observada em pesquisa simples na internet. Contudo, ao que tudo indica ainda não se pode determinar quantitativamente o uso real das colocações por pesquisa em corpora, visto que nenhuma das formas configuram nos corpora em PE e PB consultados, o CETEMPúblico e o Corpus Brasileiro, respetivamente. Desta forma, para tradução de single mother propomos mãe a solo (PE) e mãe solo (PE e PB), em detrimento de mãe solteira.

Registe-se que os neologismos em apreço passam assim a evocar outros frames: o frame Relações_pessoais que single e solteira evocam dá lugar ao frame Acompanhamento, onde se enquadram mãe a solo/mãe solo porque este compreende a situação em que "um co-participante desempenha o mesmo papel do participante em um evento ou situação", enquadrando também outras ULs como junto, em combinação, sozinho, com etc. ${ }^{27} \mathrm{Ou}$ seja, este frame abriga uma situação mais relevante à realidade do papel social de mãe no contexto das famílias monoparentais, uma vez que o frame Relações_pessoais deixou de fazer sentido. Assim sendo, estas novas expressões já não albergam valores morais e sociais sobre a estrutura da família nuclear que têm sido postos em causa no século XXI.

Quanto à correspondência ao nível linguístico e conceitual em tradução, se retomarmos a ideia de profiling ou perfilhamento mencionada anteriormente, onde o plano de fundo conceitual e o primeiro plano linguístico constituem camadas complementares do sentido lexical, podemos concluir que as traduções propostas se situam em planos de fundo distintos da UL na língua de partida - estado civil versus acompanhamento. Não obstante, o primeiro plano - o referente da UL percebido pelos interlocutores, neste caso, a mãe e chefe da família monoparental - permanece facilmente acessível e apreensível pelos falantes de PE e PB, de modo que a alteração do framing na tradução não incorre em maior processamento mental por parte do interlocutor, embora o caminho interpretativo seja alterado. Ou seja, as novas traduções propostas podem ser consideradas como equivalentes à colocação da língua de partida, nos termos do princípio da relevância de Gutt (2000), além de terem uso semelhante comprovado nas línguas de chegada, como exemplificado acima.

\section{Considerações finais}

O presente estudo, ancorado na semântica de frames, incide sobre a colocação single mother e reflete sobre as suas traduções para PB e PE, a saber, mãe a solo e mãe solo. Conforme amplamente demonstrado em Almeida (2021 - no prelo) e Almeida \& Sousa (2019), tendencialmente as formações neológicas ocorrem sobretudo na comunicação digital, que é pioneira nos novos usos linguísticos do português. Não obstante, preconiza-se que as formações em causa são motivadas por mudanças sociais observadas nas últimas décadas, que a língua natural tratou de acompanhar.

27 Traduzido pelas autoras da FrameNet de Berkeley, disponível em https://framenet2.icsi.berkeley.edu/fnReports/ data/frameIndex.xml?frame=Accompaniment. Último acesso: 04/03/2021. 


\section{Referências bibliográficas}

Almeida, M. C. (2016). Tradução versus transcriação: Abordagem cognitiva. In M. C. Almeida, L. CavacoCruz, \& I. Ramos (Eds.), Tradução, transcriação, transculturalidade (pp. 1-30). Arkonte Publishing.

- (2021, no prelo). Usos do português em meio digital: Posts de riso em análise sociopragmática. In A. Merlan, \& B. Schäfer-Priess (Eds.), Randromania im Fokus: gesprochenes Rumänisch, Portugiesisch und Galicish. Peter Lang.

Almeida, M.C.; \& Sousa, B. (2019). SMS e oralidade em português europeu. In B. Meisnitzer, \& E. Putska (Eds.), Zwischen Sprechen und Sprache/Entre fala e Lingua (pp. 321-334). Peter Lang.

Araúz, P. L.; Faber, P.; \& Montero-Martinez, S. (2012). Specialized language semantics. In P. Faber (Ed.), A Cognitive Linguistics View of Terminology and Specialized Language (pp. 95-176). Mouton de Gruyter.

Babcock, L.; \& Laschever, S. (2003). Women don't ask: Negotiation and the gender divide. Princeton University Press.

Baliana, L. K. (2013). Monoparentalidade feminina e seus desafios: Um estudo exploratório. Dissertação de Mestrado no Instituto Superior de Ciências Sociais e Políticas, Universidade de Lisboa.

Barsalou, L. D. (1999). Perceptual symbol systems. Behavioral and Brain Sciences, 22, 577-660.

Bassnett, S.; \& Lefevere, A. (Eds.). (1990). Translation, history, and culture. Cassel.

Bertoldi, A. (2010). When translation equivalents do not find meaning equivalence: a contrastive study of the frame Criminal_Process. Tese de Doutoramento não publicada, University of Texas at Austin.

Blanco-Carrión, O. (2006). Framenet as a corpus tool for the learning of second languages and for the lexical awareness of one's first language. Porta Linguarum: Revista Internacional de Didáctica de las Lenguas Extranjeras, 6, 67-76.

. (2007). La Semántica de Marcos: Análisis de "saber" según este enfoque. In C. P. López et al. Actas del VI Congreso de Lingüística General (pp. 71-81). Arco Libros.

- (2016). La semántica de marcos. In I. Ibarretxe-Antuñano, \& J. Valenzuela (Eds.), Lingüística Cognitiva (2nd Edition) (pp. 167-188). Anthropos.

Boas, H. C. (2013). Frame Semantics and Translation. In A. Rojo, \& I. Ibarretxe-Antuñano (Eds.). Cognitive linguistics and translation (pp. 125-158). De Gruyter Mouton.

Borges, L. (2020). Mãe solteira não. Mãe solo! Considerações sobre maternidade, conjugalidade e sobrecarga feminina. Revista Direito e Sexualidade, no. 1, maio de 2020.

BRASIL (2019). Mulheres comandam 40\% dos lares brasileiros. Governo do Brasil. <http://www.brasil.gov.br/ cidadania-e-justica/2015/05/mulheres-comandam-40-dos-lares-brasileiros>

Câmara, M. S. (2021). A liderança do feminino em tradução: Uma perspetiva cognitiva centrada em frames. Dissertação do Mestrado não publicada, Faculdade de Letras da Universidade de Lisboa.

De Vega, M. (1984). Introducción a la psicología cognitiva. Alianza.

Faber, P.; \& Gómez-Moreno, J. M. U. (2012). Specialized language translation. In P. Faber (Ed.), A cognitive linguistics view of terminology and specialized language (pp. 73-92). Mouton de Gruyter.

Faber, P. (2009). The cognitive shift in terminology and specialized translation. Monografías de Traducción e Interpretación, MonTI. 1. 10.6035/MonTI.2009.1.5.

Fillmore, C. J. (2006 [1982]). Frame Semantics. In D. Geeraerts (Ed.), Cognitive Linguistics: Basic readings (pp. 373-399). Mouton de Gruyter.

Fodor, J. A. (1983). The modularity of mind. MIT Press. 
Glenberg, A. M.; \& Robertson, D. A. (2000). Symbol grounding and meaning: A comparison of high-dimension and embodied theories of meaning. Journal of Memory and Language, 43, 379-401.

Gutt, E. A. (2000). Translation as interlingual interpretive use. In Venuti, L. (Ed.), The Translation studies reader (pp. 376-396). Routledge. [Originalmente publicado em 1991.]

Halverson, S. L. (2013). Implications of cognitive linguistics for translation studies. In A. Rojo, \& I. IbarretxeAntuñano (Eds.), Cognitive linguistics and translation (pp. 33-74). Mouton de Gruyter.

Harnad, S. (1987). Category induction and representation. In S. Harnad (Ed.), Categorical perception: The groundwork of cognition (pp. 535-565). Cambridge University Press. . (1990). The symbol grounding problem. Physica D., 42, 335-346.

Kussmaul, P. (1995). Training the translator. John Benjamins.

Langacker, R. (2006). Cognitive grammar. In D. Geeraerts (Ed.), Cognitive Linguistics: Basic readings (pp. 29-67). Mouton de Gruyter.

Marcus, M.; Santorini, B.; \& Marcinkiewicz, M.A. (1993). Building a large annotated corpus of English: The penn treebank. Computational Linguistics, 19, 2, 313-330.

Martín de León, C. (2013). Contribution of cognitive models of meaning to translation. In A. Rojo, \& I. Ibarretxe-Antuñano (Eds.), Cognitive linguistics and translation (pp. 99-122). Mouton de Gruyter.

Miller, L. E.; \& Miller, J. (2011). A woman's guide to successful negotiating. McGraw-Hill.

Pagano, A.; Magalhães, C. M.; \& Alves, F. (2005). Competência em tradução: cognição e discurso. Editora UFMG.

Risku, H. (2004). Translationsmanagement: Interkulturelle fachkommunikation im informationszeitalter. Gunter Narr.

Rojo, A.; \& Ibarretxe-Antuñano, I. (2013). Cognitive linguistics and translation studies: Past, present and future. In A. Rojo, \& I. Ibarretxe-Antuñano (Eds.), Cognitive linguistics and translation: Advances in some theoretical models and applications (pp. 3-30). Mouton de Gruyter.

Rojo, A. (2002). Applying frame semantics to translation: A practical example. Meta: Journal des traducteurs, XLVII, 3, 312-350. <http://doi.org/47. 312. 10.7202/008018ar>

Rosch, E. (1973). Natural Categories. Cognitive Psychology, 4, 3, 328-350. <https://doi.org/10.1016/00100285(73)90017-0>

- (1975). Cognitive representations of semantic categories. Journal of Experimental Psychology: General, 104, 3, 192-233. https:// https://doi.org/10.1037/0096-3445.104.

. (1978). Principles of categorization. In E. Rosch, \& B. B. Lloyd (Eds.), Cognition and categorization (pp. 27-48). Lawrence Earlbaum.

Ruppenhofer, J.; Ellsworth, M.; Petruck, M. R. L.; Johnson, C. R.; Baker, C. F.; \& Scheffczyk, J. (2016). FrameNet II: Extended theory and practice. <https://framenet.icsi.berkeley.edu/fndrupal/the_book>

Sandberg, S.; \& Scovell, N. (2013). Lean in: Women, work and the will to lead. Random House.

Silva, C. G.; Cassiano, K. K.; \& Cordeiro, D. F. (2019). Mãe solo, feminismo e Instagram: análise descritiva utilizando mineração de dados. XXI Congresso de Ciências da Comunicação na Região Centro-Oeste Goiânia - GO, Brasil - 22 a 24/05/2019

Steiner, G. (2000). The hermeneutic motion. In L. Venuti (Ed.), The translation studies reader (pp. 186-191). Routledge. [Originalmente publicado em 1975.]

Talmy, L. (2006). Grammatical construal: The relation of grammar to cognition. In D. Geeraerts (Ed.), Cognitive Linguistics: Basic readings (pp. 69-108). Mouton de Gruyter. 
Thompson, L. (1998). The mind and heart of the negotiator. Prentice Hall.

Wright, S. E.; \& Wright, L. (1997). Terminology management for technical translation. In S. E. Wright, \& G. Budin (Eds.), Handbook of terminology management, 1, (pp.147-159). John Benjamins.

\section{Outras Referências, Dicionários, Framenets e Corpora Consultados}

British National Corpus: https://www.english-corpora.org/bnc/

Corpus Brasileiro: https://www.linguateca.pt/acesso/corpus.php?corpus=CBRAS

Corpus CETEMPúblico (português europeu): https://www.linguateca.pt/cetempublico/

Dicionário Antônio Houaiss, versão brasileira digital monousuário 1.0. (2009). Editora Objetiva.

Dicionário Priberam Online (português europeu): https://dicionario.priberam.org/

FrameNet do International Computer Science Institute in Berkeley: https://framenet.icsi.berkeley.edu/fndrupal/

FrameNet Brasil (Laboratório de Linguística Computacional da Universidade Federal de Juiz de Fora): https://www.uff.br/framenetbr/dados/lexicon/

Glossário da FrameNet: https://framenet.icsi.berkeley.edu/fndrupal/glossary

Oxford English Dictionary Online. Disponível em https://lexico.com 